\title{
Gait Abnormality Classification in Clinical Field
}

\author{
S. Sophia, K.Sowmiya, P. Vinesha, S. Rajarajeshwari, A. Athithya Janani
}

\begin{abstract}
This paper presents a novel technique of classifying the abnormalities in gait. Determining or analyzing the different movements in the limbs of human beings, gait assessment can be made. Gait prediction plays a dynamic role in the clinical field to improve the outcome of the treatment. By predicting the type of abnormalities of gait, the patients can be provided with the proper treatment and thus improving the quality of life. In the proposed method, convolutional neural network and support vector machine algorithm have been used in classifying the various abnormalities of gait such as freezing of gait, brady kinesia, Tremor, Ataxic gait, myopathic gait and muscle atrophy. This method provides greater accuracy and thus helpful in diagnosis of various abnormalities of gait.

Keywords: Gait prediction, convolution neural network, Support vector machine, freezing of gait, Brady kinesia, Tremor, Ataxic gait, myopathic gait, muscle atrophy.
\end{abstract}

\section{INTRODUCTION}

Gait scrutiny involves a methodical learning of how a person walks and is involved in detecting the abnormalities and forecasting the treatment to people with health conditions that affects the way of walking of a person. The time period connecting first heel shit and the subsequent heel hit of identical limb constitutes one gait series. The classification of gait series is shown in Fig.1.1.

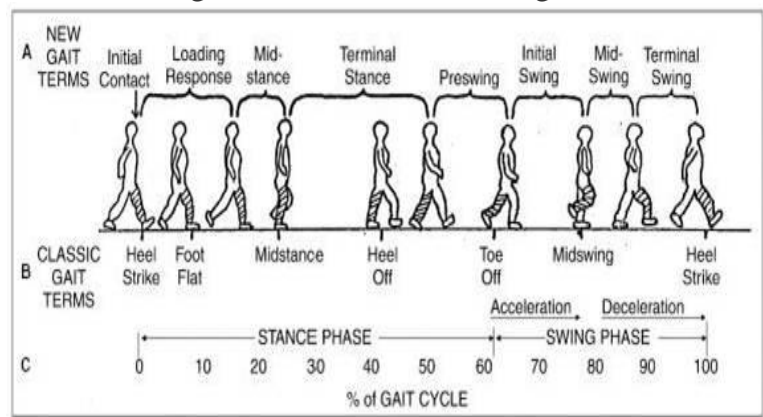

Fig. 1.1 Gait cycle showing the eight phases of gait

During the forward progression of the limbs, one of the limbs provides support while the other limb is in preparation to act as the support limb.

Stance phase originates as heel of a leg touch the base and terminates after the toe of unchanged leg move up and this phase comprises around 60 percentage of the gait sequence. Swing phase is represented as the period between

Revised Manuscript Received on March 17, 2020.

* Correspondence Author

Dr.S.Sophia*, Department of ECE, Sri Krishna College of Engineering and Technology, Coimbatore, India .Email:Sophia@skcet.ac.in

Sowmiya.K, Department of ECE, Sri Krishna College of Engineering and Technology, Coimbatore, India.Email:18epcm008@skcet.ac.in

Vinesha.P, Department of ECE, Sri Krishna College of Engineering and Technology, Coimbatore, India . Email:18epcm009@skcet.ac.in

Rajarajeshwari.S, Department of ECE, Sri Krishna College of Engineering and Technology, Coimbatore, India Email:18epcm007@skcet.ac.in

Athithya Janani.A, Department of ECE, Sri Krishna College of Engineering and Technology, Coimbatore, India Email:18epcm001@skcet.ac.in

(c) The Authors. Published by Blue Eyes Intelligence Engineering and Sciences Publication (BEIESP). This is an open access article under the CC BY-NC-ND license (http://creativecommons.org/licenses/by-nc-nd/4.0/) the toe-off of a foot and heel touch on the same foot and it comprises of about 40 percentage of the gait sequence. Swing phase is classified as acceleration, deceleration, and mid swing.

The gait sequences can be extracted using maximum sway of the shaft and they can be extracted such that gait sequence originates from maximum sway of the shaft and terminates with subsequent maximum sway of the same shaft.

Various gait forms are categorized by differences in limb-movement forms, complete speed, forces, moving and potential energy sequences, and variations in the communication with the surface. Human gaits are the numerous ways in which a human can move, either naturally or as a result of dedicated training.It is a way of walking or moving on foot.Gait analysis involves distinguishing normal and abnormal gaits.

Numerous individuals move with irregular or uneven gait sequences for long days with no signs. On the other hand, when someone has a damage or aching, ordinary gait can be reformed, follow-on anomalous walking that will lead to greater wellbeing problems such as musculoskeletal problems, cardiovascular health problems and mental health problems. Hence, gait analysis is important.

Thus, by learning the technique of how an individual walk, we are able to recognize persons' distinctive actions, govern normal gait sequences, detect problems producing ache, implement and estimate treatment to precise irregularities.

\section{LITERATURE SURVEY}

The gait analysis methods can be carried out in various aspects. According to the survey, the existing methods are enhancing to the higher level and necessary steps are also taken. The gait analysis methods now thrive as a crucial factor and an easy method of analysis. Everyone has proposed different ideas in their perspective and is being used.

Classifying the episodes of freezing of gait [1] involves Pearson's correlation that classifies the data automatically by using the parameters such as time duration, spectral mechanisms and shank movement. The Classification of FOG episodes can be achieved by means of gait parameters that are clinically acknowledged. A clinician do not need to depend upon FOG Classification output only, they can also control earlier steps of the procedure modifying the output to their individual necessities. Also, they can spot categorizations of exacting attention and acquire additional particulars regarding relations amongst gait constraints, changeability, and significances.

In biometrics VGRF can be used as a means [2] that can be attached to a current biometric arrangement for dependable proof of identity. The obtained results guarantee the utilization of unrefined information of VGRF is truthful and if it has been taken out from an ordinary subject, the

Published By:

Blue Eyes Intelligence Engineering

\& Sciences Publication

(C) Copyriaht: All riahts reserved.

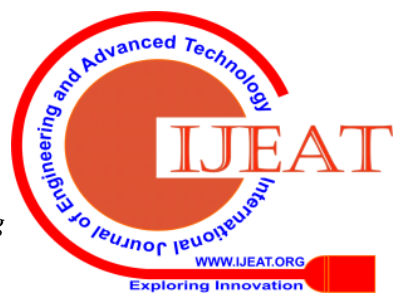


output can be weakened in abnormal gait's presence. Example include, Gait patients exaggerated with Parkinson disease reduces the accurateness in arrangement amongst subjects that concluded in elevated unfairness amongst results.

To monitor the gait pattern, Radar has newly added attention [3] in the applications such as home based safety, medicinal gait analysis, aided livelihood and restoration. Owing to its advantages such as remote, dependable and privacy-preservation detecting, Radar is capable of developing a real means for medicinal gait examination. The features of gait were obtained from parametric and nonparametric techniques for categorization.

\section{EXISTING METHOD}

Freezing of gait occurs sporadically and appears in a random manner and it was identified automatically by processing the data that was obtained from the inertial sensor. The inertial sensor was mounted on the shank part of a person and the data was collected. The data that was obtained by using the inertial sensor is processed by using the Pearson's correlation.

In this method each of the strides was described by the parameters such as time duration, spectral components and the shank displacement.

The stride that occurs in FOG patients is clearly described but it produces a discrepancy in FOG episodes. Analysing the stride cannot be processed when discrepancy occurs. This method is computationally simple and it is sensitive only to the signal shape.

Information about the intensity of the freezing of gait episodes requires further estimation. Thus a clinician need not depend on the output alone, can manage the previous steps in the algorithm according to the requirement.

\section{IV.PROPOSED WORK}

In the proposed work, the classification of abnormalities of gait is performed by using Convolution neural network classifier (CNN) and Support vector machine (SVM) algorithm. Neural network classifier is a set of algorithms that can be used for recognizing the sequences. The sensory data is read during the machine observation, categorizing or grouping the inputs. The sequences that were identified are numerical, and occur in vector form and it, should be decoded.

Neural networks are a set of procedures, demonstrated lightly afterwards the human brain, that are intended for identifying sequences. They read data by a type of mechanism awareness, classification or gathering rare input data and the outlines that are identified are mathematical, in which all actual data, such as image, sound, text or time sequences, should be decoded.

Neural networks are helpful in grouping and categorizing. They benefit to cluster unlabelled data conferring to comparations amongst the specimen input, and also order the information when the characterized dataset is taken for training. In addition neural networks produce topographies and it is given to next procedures that can be used for grouping along with arrangement.

SVM algorithm analyses the data or information that is to be classified.

Here, we perform classification of abnormalities by obtaining a gait signal from the dataset provided. The next process is the extraction of angular speed and velocity signal from the obtained gait signal. Then the movement disorders are classified by using both CNN and SVM algorithms and the performance is analysed between both of the algorithms.

The classified movement disorders include Freezing of gait, Brady Kinesia, Tremor, Ataxic gait, myopathic gait and muscle atrophy and the normal gait (not pathological gait) is also determined. Freezing of gait is the process in which the forward progression of the feet is much reduced or it is absent, Brady kinesia is a disorder in which the muscle movement is slowed and reduced, Tremor includes involuntary shaking or movement ranging from slight to severe, Ataxic gait results in abnormalities in eye movement, Myopathic gait also known as Waddling gait produces inability in standing process, Muscle atrophy is caused due to the lack of physical activities. The SVM algorithm was found to have greater accuracy then the CNN algorithm.

\section{SIMULATION RESULTS}

\section{A. Gait signal}

The obtained gait signal for the given samples is shown in Fig. 5.1

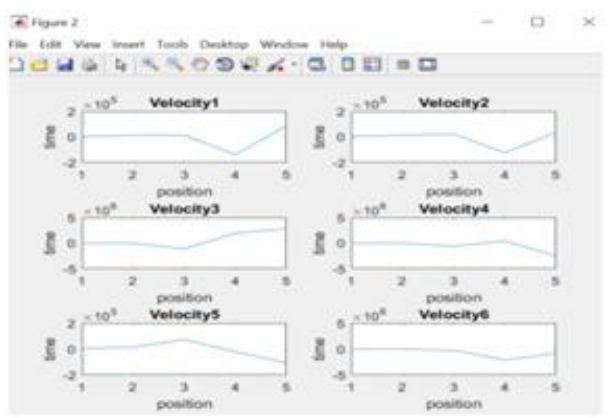

Fig. 5.1 Gait signal

The above diagram depicts the gait signals obtained from different positions of the leg.

\section{B. Velocity extraction}

Velocity extracted from the gait signal is shown in

Fig. 5.2

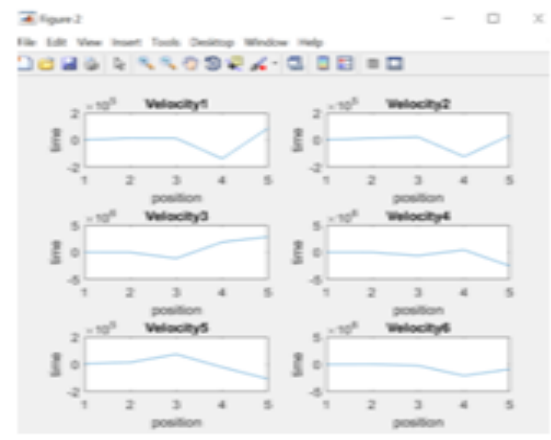

Fig. 5.2 Velocity signal

\section{Angular speed}

The angular speed extracted from the gait signal is shown in Fig. 5.3

Published By:

Blue Eyes Intelligence Engineering

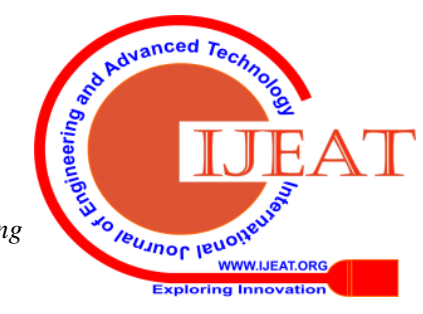




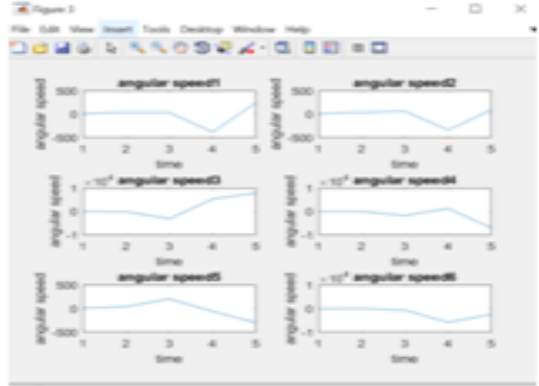

Fig. 5.3 Angular speed of the samples

\section{Accuracy and precision}

The accuracy and precision values for CNN algorithm obtained from the confusion matrix is shown in Fig. 5.4

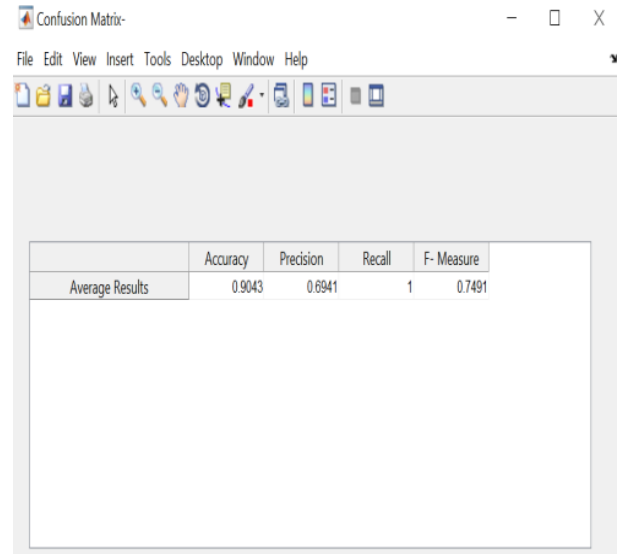

Fig. 5.4 Obtained accuracy and precision for CNN algorithm

\section{E. Confusion matrix}

The confusion matrix of CNN is shown in Fig. 5.5

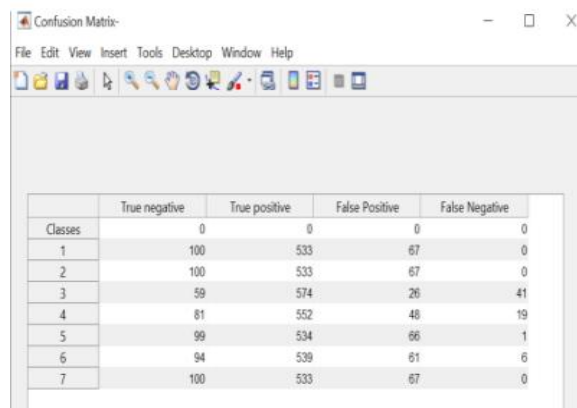

Fig. 5.5 Confusion matrix for CNN algorithm

\section{F. Accuracy and precision}

The accuracy and precision values of SVM is shown in Fig. 5.6

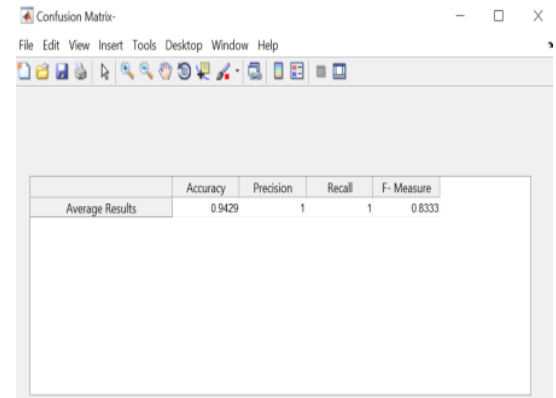

Fig. 5.6 Obtained accuracy and precision for SVM algorithm

\section{G. Confusion matrix}

The confusion matrix of SVM is shown in Fig. 5.7

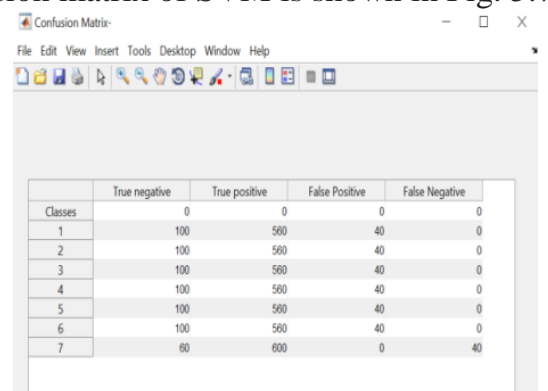

Fig. 5.7 Confusion matrix of SVM

\section{H. Abnormality classification}

Output window displaying classification of abnormalities using CNN algorithm is shown in Fig. 5.8

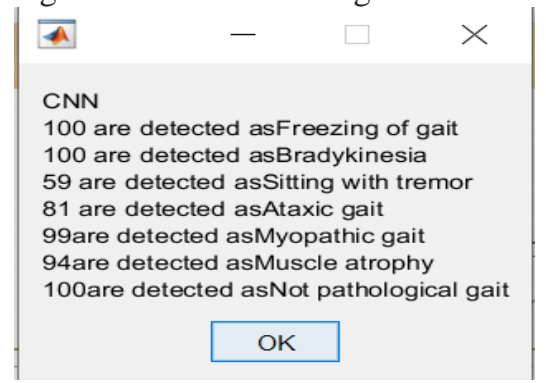

Fig. 5.8 Output screen showing the classification of abnormalities using CNN algorithm

\section{Abnormality classification:}

The output window displaying the classification of abnormalities using SVM algorithm is shown in Fig. 5.9

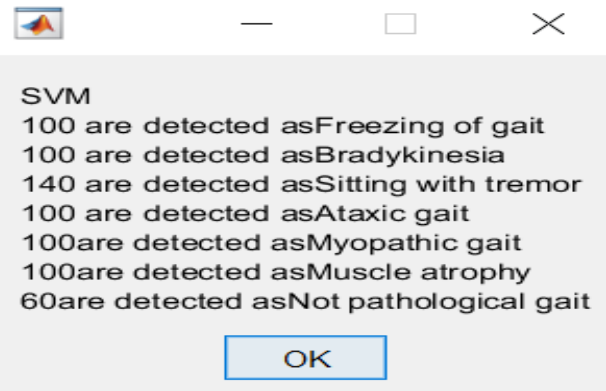

Fig. 5.9 Output screen showing the classification of abnormalities using SVM algorithm

\section{J. Comparison graph of CNN and SVM algorithm}

The comparison graph showing the performance metrics of the CNN and SVM algorithm is shown in the figure Fig.5.10

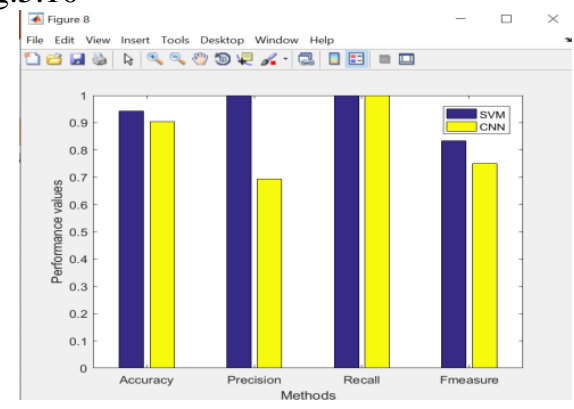

Fig. 5.10 Comparison graph

Published By:

Blue Eyes Intelligence Engineering

\& Sciences Publication

(C) Copyriaht: All riahts reserved. 


\section{VI.CONCLUSION AND FUTURE WORK}

A method to classify the abnormal conditions of the gait is proposed in this method. To classify the abnormal gaits, Convolution neural network classifier and SVM algorithm is used. By using the samples that are taken gait signal is obtained. Characteristics such as velocity, acceleration, minima and maxima, angular speed are extracted and abnormality classification is performed using both the algorithms. Accuracy and precision are also calculated by using confusion matrix. SVM algorithm is found to have greater accuracy than CNN algorithm.

In the future work, the accuracy will be further improved and remaining classifications of abnormal gait such as Rett syndrome, Huntington's disease, dystonia, Wilson's disease and Tourette syndrome will be performed.

\section{REFERENCES}

1. Milica D. Djurić-Jovičić, Nenad S. Jovičić, Saša M. Radovanović, Iva D. Stanković, Mirjana B. Popović, and Vladimir S. Kostić,' Automatic Identification and Classification of Freezing of Gai Episodes in Parkinson's Disease Patients “, IEEE transactions on neural systems and rehabilitation engineering, Vol.22, No.3, May2014

2. Rami Alkhatib, Mohamad O.Diab, Omar Itani, Camelia Chamaa, Maher Sabbah," Usage of VGRF in Biometrics: Application on Healthy and Parkinson Gaits", IEEE, 2018

3. Ann-Kathrin Seifert, Abdelhak M. Zoubir," Radar Classification of Human Gait Abnormality Based on Sum-of-Harmonics Analysis", IEEE, 2018

4. IoannisPapavasileiou*, Wenlong Zhang, Xin Wang, Jinbo Bi, Li Zhang and Song Han*," Classification of Neurological Gai Disorders Using Multi-task Feature Learning", IEEE/ACM International Conference on Connected Health: Applications, Systems and Engineering Technologies (CHASE), 2017

5. Charence Wong, Stephen McKeague, Javier Correa, Jindong Liu and Guang-Zhong Yang," Enhanced Classification of Abnormal Gait Using BSN and Depth", Ninth International Conference on Wearable and Implantable Body Sensor Networks, 2012

6. Dr.M.Pushpa Rani," Abnormal GAIT Classification Using Hybrid ELM", CCECE, 2014

7. Hany HazfizaManap, Nooritawati Md Tahir and Ahmad Ihsan M Yassin," Statistical Analysis of Parkinson Disease Gait Classification using Artificial Neural Network", IEEE, 2011

8. Xiaodong yang," Non-Contact Early Warning of Shaking Palsy", IEEE journal of translational Engineering in health care and medicine, 2019

9. Jing Gao, Peishang Gu, Qing Ren , Jinde Zhang, And Xin Song ,' Abnormal Gait Recognition Algorithm Based on LSTM-CNN Fusion Network", IEEE Access,2019

10. Qian Wang," Acceleration and Electromyography (EMG) Pattern Analysis for Children with Cerebral Palsy", IEEE $14^{\mathrm{TH}}$ International conference on E-Health Networking, applications and services, 2012

\section{AUTHORS PROFILE}

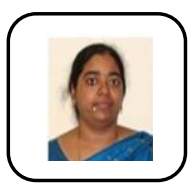

Dr. Sophia. S, Professor \& Head in the department of ECE at Sri Krishna College of Engineering and Technology. Have published a paper entitled "Probabilistic variable precision fuzzy rough set technique for discovering optimal learning patterns in e-learning “, in International Journal of Business Intelligence and Data Mining, Volume 14, Issue 1-2, Pages 121-137 in 2019 and carrying out a research project "Developing women entrepreneurs in the field of millet food production to ensure nutritional security” from 2018-2020.

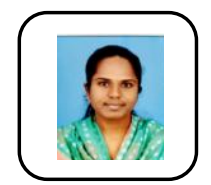

Sowmiya. K, currently pursuing a Masters degree in Engineering in the communication system stream under ECE branch at Sri Krishna College of Engineering and Technology. Have published a paper entitled "A prospective towards smart vehicle system" in e-journal Volume 7, Issue 1 0n March 2019 and "Smart bus information conveying system" in e-journal Volume 6, issue 1 in March 2018 in International Journal of Scientific Research \& development.

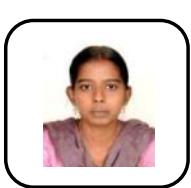

Vinesha. P, currently pursuing a Masters degree in Engineering in the communication system stream under ECE branch at Sri Krishna College of Engineering and Technology. Have published a paper entitled "A prospective towards smart vehicle system" in e-journal Volume 7, Issue 1 0n March 2019 in International Journal of Scientific Research \& development. Additionally, have presented the paper entitled "Bot for Garbage collection in cities" in the second International Conference on "Recent Advances in Engineering and Technology-ICRAET 2019” in February 2019.

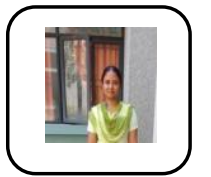

Rajarajeshwari. S, currently pursuing a Masters degree in Engineering in the communication system stream under ECE branch at Sri Krishna College of Engineering and Technology. Have published a paper entitled "A prospective towards smart vehicle system" in e-journal Volume 7, Issue 1 0n March 2019 in International Journal of Scientific Research \& development. Additionally, have presented the paper entitled "Bot for Garbage collection in cities" in the second International Conference on "Recent Advances in Engineering and Technology-ICRAET 2019 " in February 2019

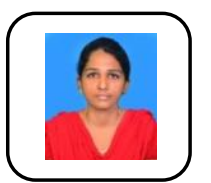

Athithya Janani. A, currently pursuing a Masters degree in Engineering in the communication system stream under ECE branch at Sri Krishna College of Engineering and Technology. Have published a paper entitled "Cognitive Technology in Various Applications" in e-journal Volume 7, Issue 1 0n March 2019 in International Journal of Scientific Research \& development.

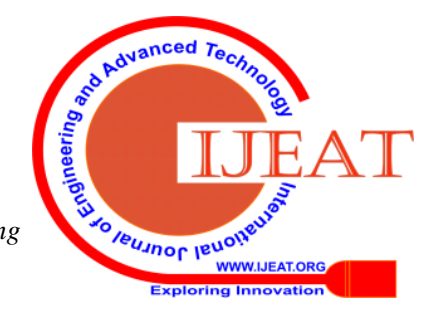

